\author{
Series A
}

\author{
I. MATHEMATICA
}

373

\title{
SETS OF ASYMPTOTIC VALUES OF POSITIVE LINEAR MEASURE
}

F. BAGEMIHL 
Communicated 9 April 1965 by P. J. Myrberg and Olli Lehto 


\section{Sets of asymptotic values of positive linear measure}

It will be shown that certain sets of asymptotic values contain sets of positive linear measure, whereas heretofore they were either known merely to contain sets of positive harmonic measure or not discussed at all. This is accomplished by generalizing a result given by Collingwood and Cartwright [3, p. 103, Lemma 4].

Let $C$ be the unit circle, $D$ be the open unit disk, and $\Omega$ be the Riemann sphere. Consider a meromorphic function $f(z)$ in $D$. The set of asymptotic values of $f$ will be denoted by $A(f)$. If $\Gamma$ is a closed subarc of $C$, then $A(f, \Gamma)$ represents the set of asymptotic values of $f$ approached along paths whose ends are contained in $\Gamma, A_{\mathfrak{p}}(f, \Gamma)$ is the set of asymptotic values of $f$ approached along paths terminating in single points of $\Gamma$, and $A_{n}(f, \Gamma)$ denotes the set of asymptotic values of $f$ approached along paths whose ends are subares of $\Gamma$, so that we have

$$
A(f, \Gamma)=A_{\mathfrak{a}}(f, \Gamma) \cup A_{\mathfrak{H}}(f, \Gamma) .
$$

If $\zeta \in C$, then $A(f, \zeta)$ means the set of asymptotic values of $f$ approached along paths terminating in $\zeta$. The set of Fatou points of $f$ on $\Gamma$ is denoted by $F(f, \Gamma)$, and $A_{F}(f, \Gamma)$ represents the set of angular limits that $f$ has at points of $\Gamma$.

By an arc at a point $\zeta \in C$ we mean a simple continuous curve $\Lambda: z=z(t) \quad(0 \leqq t<1) \quad$ such that $\quad|z(t)|<1 \quad$ for $0 \leqq t<1 \quad$ and $\lim z(t)=\zeta$. If $0 \leqq t_{0}<1$ and $z\left(t_{0}\right)=\zeta_{0}$, then the arc $A^{\prime}: z=z(t)$ $t \rightarrow 1$ $\left(t_{0} \leqq t<1\right)$ is called the terminal subarc of $\Lambda$ determined by $\zeta_{0}$.

We shall be concerned with the following

Hypothesis $\mathfrak{H}$. (i) $f(z)$ is a meromorphic function in $D$;

(ii) $\Gamma$ is a closed subarc of $C$ with distinct end points $\zeta_{1}$ and $\zeta_{2}$;

(iii) $J$ is a Jordan arc lying, except for its end points $\zeta_{1}$ and $\zeta_{2}$, in $D$;

(iv) $G$ is the subregion of $D$ whose frontier is $J \cup \Gamma$;

(v) there exist distinct finite values $\omega_{1}$ and $\omega_{2}$ such that

$$
\lim _{\substack{z \rightarrow \zeta_{1} \\ z \in J}} f(z)=\omega_{1}, \quad \lim _{\substack{z \rightarrow-2 \\ z \in J}} f(z)=\omega_{2} ;
$$

(vi) $f$ has no asymptotic path in $G$ on which $f$ tends to $\infty$.

Research sponsored by the Mathematics Research Center, United States Army, Madison, Wisconsin, under Contract No.: DA-11-022-ORD-2059. 
Our basic result is

Theorem 1. Assume $\mathfrak{H}$ and that $f$ is holomorphic in $G$. Then $A_{\mathfrak{p}}(f, \Gamma)$ contains a set of positive linear measure.

Proof: The arc $J$ minus its end points will be called $J_{0}$. Take a point $z_{0}$ on $J_{0} ; z_{0}$ divides $J_{0}$ into an arc $\Lambda_{1}$ at $\zeta_{1}$ and an are $\Lambda_{2}$ at $\zeta_{2}$. Denote by $L$ the open rectilinear segment joining $\omega_{1}$ and $\omega_{2}$. Choose an arbitrary point $\mu \in L$ and consider the straight line $M$ perpendicular to $L$ and containing $\mu$. Let $\delta$ be a positive number smaller than the distance between $M$ and the set $\left\{\omega_{1}, \omega_{2}\right\}$. Then therc exist terminal subarcs $\Lambda_{1}^{\prime}, \Lambda_{2}^{\prime}$ of $\Lambda_{1}, \Lambda_{2}$, respectively, such that

$$
\left|f(z)-\omega_{1}\right| \leqq \delta \quad\left(z \in \Lambda_{1}^{\prime}\right), \quad\left|f(z)-\omega_{2}\right| \leqq \delta \quad\left(z \in A_{2}^{\prime}\right)
$$

Since the set of zeros of $f^{\prime}(z)$ in $G$ is isolated, we can find a circular arc $K$ with center at the origin and lying in $G$, with one end point $\lambda_{1}$ on $\Lambda_{1}^{\prime}$ and the other end point $\lambda_{2}$ on $\Lambda_{2}^{\prime}$, such that $f^{\prime}(z) \neq 0$ on $K$. Since $f$ is also holomorphic on $K, K$ is mapped by $w=f(z)$ onto a bounded simple analytic arc $T$, and $T$ intersects $M$ at least once, but only a finite number of times. Thus either (a) $T \cap M$ consists of a single point $p$, or else (b) there are distinct points $p_{1}, p_{2}$ in $T \cap M$ such that any other point in $T \cap M$ lies on $M$ between $p_{1}$ and $p_{2}$. Let $G_{0}$ be the subregion of $G$ whose frontier consists of $\Gamma, K$, the terminal subarc $\Lambda_{1}^{\prime \prime}$ of $\Lambda_{1}^{\prime}$ determined by $\lambda_{1}$, and the terminal subarc $\Lambda_{2}^{\prime \prime}$ of $\Lambda_{2}^{\prime}$ determined by $\lambda_{2}$.

There is an ordinary element of the inverse $f^{-1}(w)$ of the function $f(z)$ in case (a) at $p$ and in case (b) at $p_{1}$ and at $p_{2}$. In case (a) this element can be continued towards $\infty$ in either direction along $M$ without encountering another point of $T$, and in case (b) the elements at $p_{1}$ and $p_{2}$ can be continued towards $\infty$ in suitable directions so as not to encounter another point of $T$. In case (a), a direction of continuation can be chosen, and in case (b) one of the two points $p_{1}, p_{2}$ can be chosen, so that the curve $m$ on which the appropriate path of eontinuation along $M$ is mapped lies in $G_{0}$. The continuation in question cannot be made to the point $\infty$ because $\infty$ is neither an assumed value nor an asymptotic value of $f(z)$ in $G_{0}$. Consequently there is a finite point $q$ on $M$ such that either the continuation has a boundary element (see [3, p. 100]) at $q$ or the continuation is terminated by a transcendental singularity at $q$. The curve $m$ is consequently an asymptotic path of $f(z)$ which either terminates in a point of $\Gamma$ or has its end on $\Gamma$, and on which $f(z) \rightarrow q$ as $|z| \rightarrow 1$. Hence $q \in A(f, \Gamma)$.

Now consider $f(z)$ restricted to $G_{0}$. According to Kierst [8], $A(f$, $\left.\Gamma \cup \Lambda_{1}^{\prime \prime} \cup K \cup \Lambda_{2}^{\prime \prime}\right)$ is an analytic subset of $\Omega$. Evidently $A\left(f, \Lambda_{1}^{\prime \prime} \cup K \cup \Lambda_{2}^{\prime \prime}\right)$ $=A_{\mathfrak{p}}\left(f, \Lambda_{1}^{\prime \prime} \cup K \cup A_{2}^{\prime \prime}\right)$ is an $F_{\sigma}$. Now $A_{\mathfrak{u}}(f, \Gamma)$ contains at most enumer- 
ably many finite values, because otherwise, as was pointed out to me by J. E. McMillan, there would exist two such values with corresponding asymptotic paths which meet on $\Gamma$, and this is readily seen to contradict (vi); let us assume that $\mu$ was chosen so that $q \notin A_{\mathfrak{u}}(f, \Gamma)$. Then $A_{\mathfrak{p}}(f$, $\left.\Gamma \cup \Lambda_{1}^{\prime \prime} \cup K \cup \Lambda_{2}^{\prime \prime}\right)$ is also an analytic set. Consequently [5, p. 370, 42 $\left.\cdot 1 \cdot 3\right]$ the set

$$
E \equiv A_{\Downarrow}\left(f, \Gamma \cup \Lambda_{1}^{\prime \prime} \cup K \cup \Lambda_{2}^{\prime \prime}\right)-A_{\Downarrow}\left(f, \Lambda_{1}^{\prime \prime} \cup K \cup \Lambda_{2}^{\prime \prime}\right)
$$

is an analytic set; it is clearly a subset of $A_{\mathrm{v}}(f, \Gamma)$ and contains $q$. The set $E$ is Carathéodory linearly measurable [6, p. 83, 7. 1. 211 and p. 84, Bibliography; p. 105, 8. 5. 1]. Since $\mu$ was an arbitrary point of $L$ with at most enumerably many exceptions, the orthogonal projection of $E$ onto $L$ contains almost all of $L$, and hence [13, p. 533] $E$ is of positive linear measure. This completes the proof.

In Theorem 1 we may not drop the hypothesis that $f$ be holomorphic in $G$. This is evident from

Theorem 2. Under the assumption $\mathfrak{\mathfrak { H }}$, it is possible to have $A(f)=\left\{\omega_{1}, \omega_{2}\right\}$.

Proof: We take $\zeta_{1}=+1, \zeta_{2}=-1 ; \Gamma$ to be the semicircle $|z|=1, \Im(z) \geqq 0 ; J$ to be the segment $-1 \leqq z \leqq+1$; and finally $\omega_{1}=+1, \omega_{2}=-1$. Define $B_{0}$ to be the arc $\frac{1}{2} \leqq z<1$ and $B_{1}$ to be the arc $-1<z \leqq-\frac{1}{2}$; and for $n=1,2,3, \ldots$, let

$$
J_{n}=\left\{z:|z|=\frac{n+1}{n+2}\right\} .
$$

We put

$$
S=B_{0} \cup B_{1} \cup\left(\bigcup_{n=1}^{\infty} J_{n}\right)
$$

and call $S$ the skeleton.

We now define a continuous function $g(z)$ on $S$. Let

$$
g(z) \equiv+1\left(z \in B_{0}\right), \quad g(z) \equiv-1\left(z \in B_{1}\right) .
$$

Define $C^{+}\left(C^{-}\right)$to be the subarc of $C$ on which $\widetilde{\mho}(z) \geqq 0(\widetilde{\mho}(z) \leqq 0)$, and for every $n$, let $J_{n}^{+}\left(J_{n}^{-}\right)$be the subarc of $J_{n}$ on which $\Im(z) \geqq 0$ $(\widetilde{\mho}(z) \leqq 0)$. For $n=2 k-1(k=1,2,3, \ldots)$, define $g(z)$ on $J_{n}$ as follows: $g(z)$ is a homeomorphism of $J_{n}^{-}$onto $C^{-}$and a homeomorphism of $J_{n}^{+}$onto $C^{-}$. For $n=2 k(k=1,2,3, \ldots)$, define $g(z)$ to be a homeomorphism of $J_{n}^{-}$onto $C^{+}$and a homeomorphism of $J_{n}^{+}$onto $C^{+}$. Clearly $g(z)$ is defined and continuous on $S$. that

According to [1], there exists a meromorphic function $f(z)$ in $D$ such 


$$
\lim _{\substack{|z| \rightarrow 1 \\ z \in S}}|f(z)-g(z)|=0 .
$$

It is evident from this and the definition of $g(z)$ that $\mathfrak{H}$ is satisfied but $A(f)=\{+1,-1\}$.

Theorem 3. Let $f(z)$ be holomorphic and not constant in D. Suppose that $B$ is a subarc of $C, E$ is a subset of $B$ of positive measure, and $f$ has an angular limit at every point of $E$, but $\infty \notin A(f, B)$. Then $A_{\mathfrak{p}}(f, B)$ contains a set of positive linear measure.

Proof: A theorem of Priwalow [12, p. 210] implies that there exist points $\zeta_{1}, \zeta_{2}$ in $E$ and finite values $\omega_{1}, \omega_{2}$ such that $f$ has the angular limit $\omega_{1}, \omega_{2}$ at $\zeta_{1}, \zeta_{2}$, respectively. Let $\Gamma$ be the subarc of $B$ with end points $\zeta_{1}, \zeta_{2}$, and let $J$ be the union of the radii at $\zeta_{1}$ and $\zeta_{2}$. Then the hypothesis of Theorem 1 is satisfied, and the conclusion of Theorem 3 follows from that of Theorem 1 .

Remark. The assumption $\infty \notin A(f, B)$ is necessary in Theorem 3 even if $f$ is of bounded characteristic and the measure of $E$ equals the length of $B$ : see [4, p. 98].

Theorem 4. Assume $\mathfrak{H}$ and that $f$ is holomorphic and normal in $G$. Then $A_{F}(f, \Gamma)$ is of positive linear measure.

Proof: The set $F(f, \Gamma)$ is a Borel set [7, p. 275], so that $A_{F}(f, \Gamma)$ is an analytic set $[7$, p. 269] and hence is linearly measurable. Moreover, by [2, p. 15, Theorem 3], $F(f, \Gamma)$ is of positive measure because of (vi). According to $\left[9\right.$, p. 53, Theorem 2], $A_{p}(f, \Gamma)=A_{F}(f, \Gamma)$. The conclusion of Theorem 4 now follows from that of Theorem 3 .

Corollary. Let $f(z)$ be a nonconstant normal holomorphic function in $D$. Suppose that $B$ is a subarc of $C$ for which $\infty \notin A(f, B)$. Then $A_{F}(f, B)$ is of positive linear measure.

MacLane [10] has defined $\mathscr{A}$ to be the class of nonconstant holomorphic functions in $D$ having asymptotic values at each point of an everywhere dense set of points on $C$.

Theorem 5. Let $f \in \mathscr{A}$. Suppose that $B$ is a subarc of $C$ for which $\infty \notin A_{\mathrm{n}}(f, B)$. Then $A_{\mathrm{u}}(f, B)$ contains a set of positive linear measure.

Proof: According to [10, p. 28, Corollary], $A(f, B)$ contains a closed set $H$ of positive harmonic measure. If $\omega_{1}$ and $\omega_{2}$ are distinct finite values in $H$, then $\left[10\right.$, p. 18 , Theorem 4] $\left\{\omega_{1}, \omega_{2}\right\} \subset A_{\mathfrak{p}}(f, B)$. The conclusion now follows from Theorem 1 .

Theorem 6. Let $f(z)$ be a nonconstant holomorphic function in $D$. Suppose that $f$ has only finitely many asymptotic tracts for $\infty$, and the ends of the arc tracts of $f$ for $\infty$ do not cover $C$. Then $A_{p}(f)$ is of positive linear measure.

Proof: The references to [8] and [6] in the proof of Theorem 1 show 
that the set $A(f)$ is linearly measurable. McMillan has shown [11, Theorem 2] that there exists a subset $E$ of $C$ of positive measure such that $f$ has a finite asymptotic value at each point of $E$, and [11, Theorem 4] the set of these asymptotic values contains a closed set of positive harmonic measure. It is readily seen that $\mathfrak{H}$ is satisfied for a suitable $\Gamma$, and application of Theorem 1 completes the proof.

\section{References}

[1] BAGeminL, F.: Characterization of the set of values approached by a meromorphic function on sequences of Jordan curves - Ann. Acad. Sci. Fennicæ A I 328 (1963), $1-14$.

[2] - - and Seidel, W.: Koebe arcs and Fatou points of normal functions Comment. Math. Helv. 36 (1961), 9-18.

[3] Collingwood, E. F., and CARTwright, M. L.: Boundary theorems for a function meromorphic in the unit circle - Acta Math. 87 (1952), 83-146.

[4] Frostman, O.: Potentiel d'équilibre et capacité des ensembles avec quelques applications à la théorie des fonctions - Meddel. Lunds Univ. Mat. Sem. 3 (1935), 1-118.

[5] Hahn, H.: Reelle Funktionen - Leipzig, 1932.

[6] -»- and Rosenthal, A.: Set functions - Albuquerque, 1948.

[7] Hausdorff, F.: Mengenlehre - Berlin, 1935.

[8] KIERST, S.: Sur l'ensemble des valeurs asymptotiques d'une fonction méromorphe dans le cercle-unité - Fund. Math. 27 (1936), 226-233

[9] Lehto, O., and Virtanen, K. I.: Boundary behaviour and normal meromorphic functions - Acta Math. 97 (1957), 47-65.

[10] MacLane, G. R.: Asymptotic values of holomorphic functions - Rice Univ. Studies 49 (1963), 1-83.

[11] McMillan, J. E.: Asymptotic values of functions holomorphic in the unit disc Michigan Math. J. 12 (1965), $141-154$.

[12] Priwalow, I. I.: Randeigenschaften analytischer Funktionen - Berlin, 1956.

[13] RANDolPh, J. F.: Carathéodory measure and a generalization of the GaussGreen lemma - Trans. Amer. Math. Soc. 38 (1935), 531-548.

\section{University of Wisconsin-Milwaukee}

